\title{
Single Orbital Metastasis in Castration-Resistant Prostate Cancer
}

\author{
Sonia García-Cabezas ${ }^{1}$, Maria del Carmen Moreno-Manzanaro Moreno', \\ "Amalia Palacios-Eito ${ }^{1}$ \\ ${ }^{1}$ Department of Radiation Oncology, Reina Sofia University Hospital, Cordoba, \\ Spain \\ *Corresponding author: Amalia Palacios-Eito, Department of Radiation \\ Oncology, Reina Sofia University Hospital, Avda. Menéndez Pidal s/n, 14004, \\ Cordoba, Spain. Tel: +34957011647; Fax: +34957011414; E-mail: amalia. \\ palacios.sspa@juntadeandalucia.es
}

DOI: $10.30699 /$ mci.4.4.363

Submitted: 14 May 2020

Revised: 21 July 2020

Accepted: 3 September 2020

e-Published: 1 October 2020

Keywords:

Prostatic Neoplasms

Orbital Neoplasms

Neoplasm Metastasis
Introduction: Orbital metastasis of prostate cancer (PC) is very rare and even more unique in castration-resistant PC (CRPC). In this scenario, choline positron emission tomography/computed tomography (choline PET/CT) is the gold-standard restaging method of choice available in our setting, and new anti-androgens treatments show improvement in overall survival.

Case presentation: We report the case of a 69-year-old male patient diagnosed with PC, treated with radical prostatectomy, and salvage radiotherapy after biochemical recurrence. After new prostate-specific antigen (PSA) progression, androgen deprivation therapy (ADT) was started. Four and a half years later, and already labeled as non-metastatic CRPC with a negative extension study, including choline PET/CT, he developed an accidental left frontal head trauma, presenting with proptosis, palpebral oedema, and oculomotor disorder. Computed tomography (CT) and magnetic resonance imaging (MRI) showed a destructive bone lesion in the left orbit, associated with a soft tissue mass. These findings, suggestive of a neoplastic lesion, were histologically confirmed PC metastasis. Treatment was initiated with abiraterone, with a rapid improvement of symptoms, a progressive decrease of PSA, and a significant radiological response.

Conclusion: Orbital metastases may present with proptosis and should be considered in patients with a history of cancer. If the ocular-orbital disease is suspected, the nuclear medicine physician should be aware that the choline PET/CT imaging includes the orbits. Tolerability and response to treatment with abiraterone were excellent.

(C) 2020. Multidisciplinary Cancer Investigation

\section{INTRODUCTION}

Orbital metastases of solid tumors are quite rare, developing in $2 \%-5 \%$ of the patients with cancer. In $25 \%$ of the patients with ocular or orbital metastases, they are the first presenting sign of the disease [1, $2]$. Breast cancer is the most common tumor, with prostate cancer (PC) occupying the second or third position (3\%-10\%), according to the different published series $[1,3]$. Signs at diagnosis are usually non-specific. Proptosis is the main finding. Other signs include oculomotor disorder, inflammation, pain, and sometimes the appearance of a mass [1]. Imaging characteristics are also non-specific, and computed tomography (CT) and magnetic resonance imaging (MRI) are the main exams to evaluate orbital lesions [1]. However, definitive diagnosis requires histological confirmation [2]. The therapeutic strategy is established based on tumor hormone sensitivity, extent and burden 
of disease, oligo- or polymetastatic disease, as well as comorbidity and patient preferences [1, 4]. In recurrent castration-resistant (CR) PC, choline positron emission tomography/computed tomography (choline PET/CT) is the restaging method of choice in our setting, especially when traditional $\mathrm{CT}$ and bone scan studies are negative [5]. Radiotherapy is the mainstay treatment for orbital metastases, and androgen deprivation therapy (ADT) plays a pivotal role in the management of metastases from hormone-sensitive tumors, such as PC [1]. Different drugs are effective in CRPC, including the combination of abiraterone acetate and prednisone, with an improvement in median radiographic progression-free survival from 8 to 16 months [6], and in median overall survival from 30.3 to 34.7 months [7] compared with the combination of prednisone and placebo. Eye enucleation, although rarely used, is indicated in patients with ocular pain and complete vision loss [8]. We report an unusual case of a patient diagnosed with CRPC and a single orbital metastasis, unnoticed after choline PET/CT, treated with abiraterone acetate.

\section{CASE PRESENTATION}

We present the case of a 69 -year-old male patient diagnosed with low-risk PC in 2010. Initially, he was under active surveillance for one year. Due to histological progression, with a Gleason score of 7 $(4+3)$ and a prostate-specific antigen (PSA) level of $5.6 \mathrm{ng} / \mathrm{mL}$, a radical prostatectomy was performed. Pathological examination of the surgical specimen indicated prostate adenocarcinoma, pT3apN0, and the Gleason score of $7(4+3)$. Thirteen months later, with a PSA level of $0.91 \mathrm{ng} / \mathrm{mL}$, the patient was referred to our department with a diagnosis of biochemical recurrence, which was treated with salvage radiotherapy. A total dose of 70 Gy was administered to the prostate bed using the volumetric modulated arc therapy technique, reaching a nadir of $0.27 \mathrm{ng} / \mathrm{mL}$. Eighteen months after irradiation, a new biochemical progression was detected $(\mathrm{PSA}=1.44 \mathrm{ng} / \mathrm{mL}$ ), with a rapid increase to $8.15 \mathrm{ng} /$ $\mathrm{mL}$ three months later. A thoraco-abdominopelvic CT scan found no lesions, and a bone scan only found focal increased uptake in the left orbit, which was difficult to assess and coincided in time with a left blepharoconjunctivitis under treatment by his primary care physician (Figure 1).

He started treatment with ADT in March 2014,
Figure 1: Bone Scan Showing a Focal Increased Uptake in the Left Orbit

with a quarterly follow-up and PSA $<1 \mathrm{ng} / \mathrm{mL}$ until September 2018, when it rose to $1.98 \mathrm{ng} / \mathrm{mL}$. Five months before, he had undergone surgery for the right eye cataract and had left vision deficit. $\mathrm{He}$ was diagnosed, by his ophthalmologist, with the left eye cataract and significant Fuchs' dystrophy. A new extension study by $\mathrm{CT}$ was requested, with no evidence of distant disease, and a bone scan showed persistent increased left orbital uptake with no changes reported as a lesion of probable nonneoplastic origin. The $18 \mathrm{~F}$-choline PET/CT also did not find any lesions suggesting malignancy. Two weeks before, he had undergone the left phakectomy, and was awaiting endothelial cornea transplantation. The patient continued to be asymptomatic, with follow-up every three months and progressive PSA elevation with a doubling time $<6$ months. With a PSA level of $12.40 \mathrm{ng} / \mathrm{mL}$, he was evaluated by the Urology Department. Another choline PET/ CT was performed, which was again negative for neoplastic activity. After the development of an accidental direct trauma in the left frontal region, the patient went to the emergency room. The ophthalmological examination revealed proptosis, a 
hard, firm, adherent tumor of the left pterion, with the invasion of the external orbital wall and eyelid oedema. External movements of the left eye were affected and caused the restriction of supraversion and external adduction movements (Figure 2).

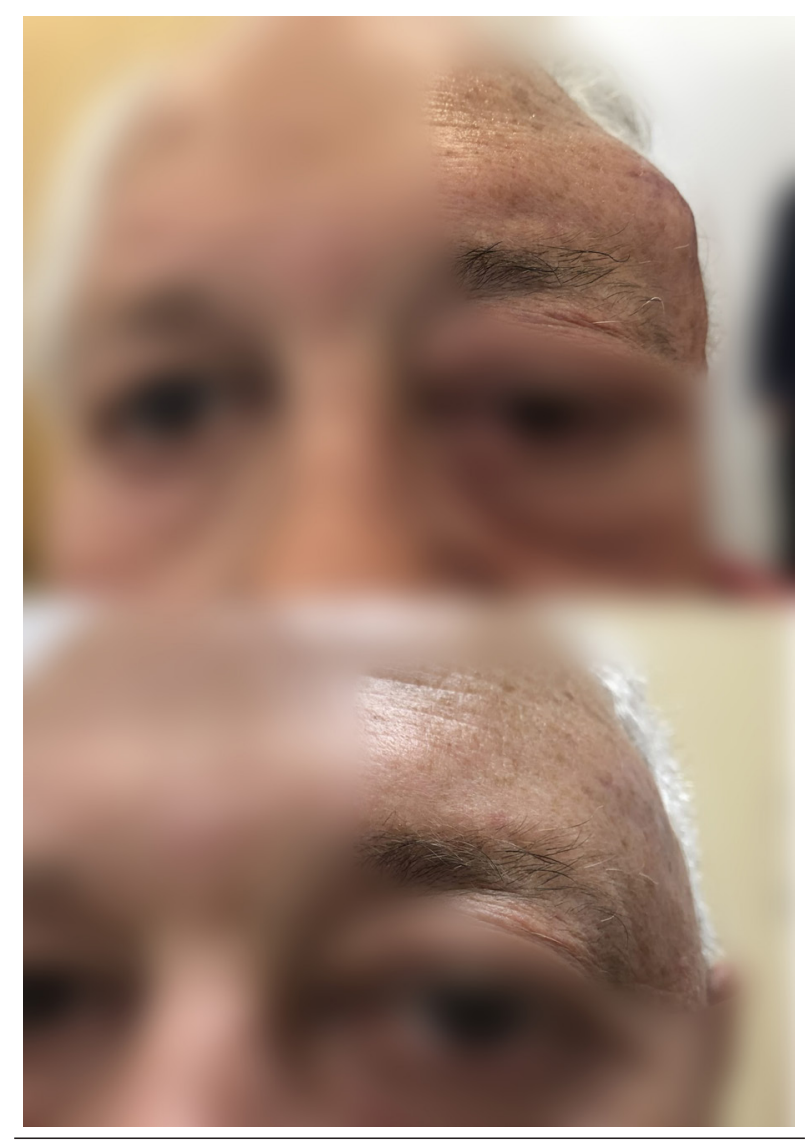

Figure 2: Patient Image at Diagnosis of Orbital Metastasis and One Month After Initiate With Abiraterone

The brain CT performed in the emergency room revealed a destructive bone lesion in the lateral wall and roof of the left orbit, with a soft tissue mass inserted into the orbit and intracranial extra-axial space, suggesting a neoplastic lesion (Figure 3).

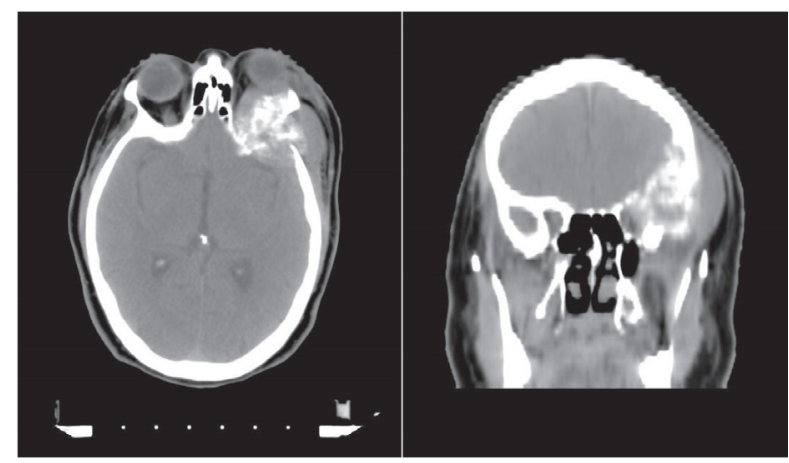

Figure 3: Brain CT Scan Showing Destructive Bone Lesion With Soft Tissue Mass
The incisional biopsy of the lesion reported a metastasis of acinar adenocarcinoma of the prostate. The MRI of the orbit showed a large mass of aggressive appearance, centered on the left sphenoid wing, which caused extensive bone destruction and extended into the orbit, causing displacement of extrinsic muscle groups and the eyeball. The mass extended to extracranial soft tissue and typically to the cerebral extra-axial compartment in the left frontal region, with associated dural uptake, measuring $54 \times 52 \times 53 \mathrm{~mm}$ in the craniocaudal and transverse antero-posterior diameters, respectively (Figure 4).

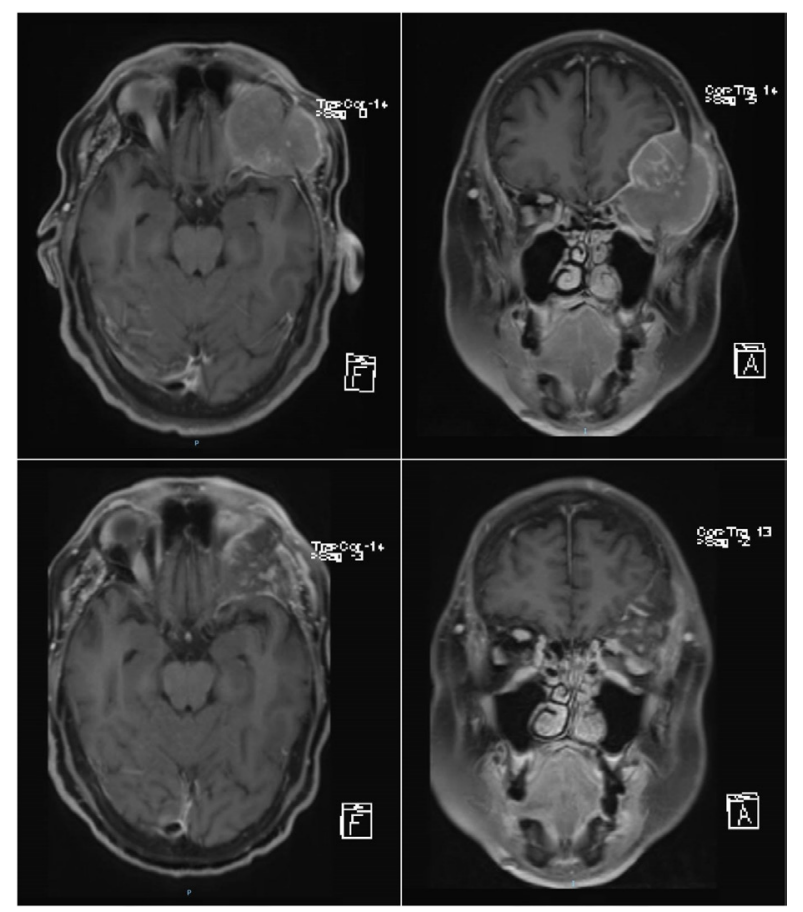

Figure 4: MRI Showing an Aggressive Mass With Bone Destruction And Invasion Of Orbit

MRI after treatment with abiraterone, with significant reduction of bone and soft tissue component involvement

Based on the diagnosis of single orbital metastasis of CRPC, treatment was started with abiraterone acetate, with marked clinical improvement of orbital symptoms several weeks later, and progressive PSA decrease in monthly laboratory tests. In the followup MRI five months after the start of treatment, a significant decrease was observed in the size of bone involvement and soft tissue component using RECIST (response evaluation criteria in solid tumors) criteria (Figure 4). The patient is currently under treatment with abiraterone and remains clinically stable, with the last PSA level of $0.21 \mathrm{ng} / \mathrm{mL}$. 


\section{DISCUSSION}

PC usually metastasizes to pelvic nodes and the axial skeleton. Intracranial metastases are uncommon. Long and Husband [9] retrospectively analyzed CT scans from 508 patients with PC to document the presence or absence of uncommon metastases. The orbit was the second most common area of unusual PC metastasis, with atypical lymph node metastasis being the first. Although PC may present with metastasis to the orbital or ocular region, it usually develops in the setting of diffuse metastatic disease [3]. Lefresne et al, [10] reported that of 48 cases of orbital and ocular metastases secondary to PC published since 1975, 38\% were the first manifestation of the disease. There were two interesting aspects in the present case: single orbital metastasis in CRPC, and that it was not identified despite close follow-up.

\section{Clinical Presentation, Diagnosis, and Treatment}

Clinical presentation depends on the structures involved. Specifically, in this case, the diagnosis was made after performing an emergency head CT for accidental trauma. The patient had vision loss justified by significant Fuchs' dystrophy and was awaiting endothelial corneal transplantation. These circumstances masked the symptoms of the orbital lesion. Retrospectively studying the case, he already had a difficult to assess increased scintigraphic uptake in the left orbit after biochemical progression subsequent to salvage radiotherapy. Therefore, the metastasis probably existed at this time and was not detected since it coincided with an infection of the left eye. Currently, the EAU-ESTRO-ESURSIOG guidelines on prostate cancer recommend performing a choline $\mathrm{PET} / \mathrm{CT}$ in patients with PSA $>1 \mathrm{ng} / \mathrm{mL}$ after radical prostatectomy [5]. However, when the patient exceeded this number, it was not yet the standard practice. Concerning the average time between the diagnosis of $\mathrm{PC}$ and the occurrence of orbital metastasis, in the series published by Long and Husband [9], it was 3.7 years (ranges 1-8). In the current case, if the time when the diagnosis confirmation is considered, it took nine years. If the time from metastasis by increased scintigraphic uptake is considered, it was four years. Radiologically, orbital metastases from PC are usually osteoblastic, unlike other tumours manifested as lytic bone lesions or soft tissue masses
$[1,11]$. However, lymphomas and meningiomas may have the same characteristics [12]. This different presentation and the unusual site of metastasis make histological confirmation highly advisable.

Choline PET/CT is the restaging method of choice in our setting for biochemical recurrence of PC. It is standard practice when performing PET/CT, regardless of the employed radiotracer, to set the upper limit of the scan below the orbits, in order to reduce the dose received by the lenses and end the scan in the upper third of the thighs. It was the reason for the delay in diagnosis in the patient, associated with the dismissal of increased scintigraphic uptake due to overlapping ocular disease. This case is discussed with nuclear medicine specialists, and an alert is established that if the orbital disease is suspected, it should be reported when requesting the study. Gallium Ga 68 (68Ga)-labelled prostatespecific-membrane antigen (PSMA) PET/TC and PET/MRI demonstrate higher detection rates than the conventional imaging modalities. Both methods are the gold standards for restaging recurrent $\mathrm{CP}$ in the countries where such imaging modalities are available [13]. However, none of them were performed on the patient, since in our setting, they were not available due to their high cost. In patients with metastatic CRPC (mCRPC), different systemic treatments show improvements in overall survival, including the chemotherapeutic agents, docetaxel and cabazitaxel, androgen-targeting agents, such as abiraterone and enzalutamide, a vaccine (sipuleucel-T), and a radiodrug (radium-223) [14]. However, mCRPC remains an incurable disease associated with a life expectancy of 2-3 years [4]. Different studies report, specifically in orbital metastases, a survival of 7.5-30 months [15]. In different published articles, hormone therapy results in an improvement in symptoms and proptosis [12, 15]. In the current case, the patient was already $\mathrm{CR}$; therefore, it was decided to start treatment with abiraterone acetate. To the best of authors knowledge, it was the first published case of single orbital metastasis from PC treated with this drug. To date (eight months from the start of abiraterone), the patient improved clinically and has a PSA level that is still decreasing. Although radiotherapy is extensively effective in the treatment of orbital metastases, with reduction of tumor size and improvement of vision, given the good response to 
treatment with abiraterone, it was decided to wait and delay its administration due to the expected potential toxicity in the form of retinopathy and neuropathy, since the mass was compressing the optic nerve.

In conclusion, orbital metastasis may present with proptosis and should be considered in patients with a history of cancer. It is advisable to inform nuclear medicine specialists about the suspected ocular-orbital disease to include this area in the imaging acquisition. In the current case, tolerability and response to treatment with abiraterone were excellent.

\section{ACKNOWLEDGEMENTS}

Not applicable

\section{CONFLICTS OF INTEREST}

The authors declared no conflict of interest.

\section{ETHICS APPROVAL}

The written informed consent was obtained from the patient to publish the information and images.

\section{REFERENCES}

1. Ahmad SM, Esmaeli B. Metastatic tumors of the orbit and ocular adnexa. Curr Opin Ophthalmol. 2007;18(5):405-13. DOI: $10.1097 / I C U .0 b 013 e 3282 c 5077 \mathrm{c}$ PMID: 17700235.

2. Goldberg RA, Rootman J, Cline RA. Tumors metastatic to the orbit: a changing picture. Surv Ophthalmol. 1990;35(1):1-24. DOI: 10.1016/0039-6257(90)90045-W PMID: 2204127.

3. Shields JA, Shields CL, Brotman HK, Carvalho C, Perez N, Eagle RC, Jr. Cancer metastatic to the orbit: the 2000 Robert M. Curts Lecture. Ophthalmic Plast Reconstr Surg. 2001;17(5):346-54. DOI: 10.1097/00002341-20010900000009 PMID: 11642491.

4. Palacios-Eito A, Bejar-Luque A, Rodriguez-Linan M, Garcia-Cabezas S. Oligometastases in prostate cancer: Ablative treatment. World J Clin Oncol. 2019;10(2):38-51. DOI: $10.5306 /$ wjco.v10.i2.38 PMID: 30815370 .

5. Mottet N, van den Bergh RCN, Briers E, Bourke L, Cornford P, De Santis M, et al. EAU - ESTRO - ESUR - SIOG Guidelines on Prostate Cancer. The Netherlands: European Association of Urology; 2018 [updated 2018 Nov 12; cited
2017 Nov 12,]. Available from: https://www.partnershipagainstcancer.ca/db-sage/sage20181112/\#.

6. Ryan CJ, Smith MR, de Bono JS, Molina A, Logothetis $\mathrm{CJ}$, de Souza P, et al. Abiraterone in metastatic prostate cancer without previous chemotherapy. N Engl J Med. 2013;368(2):138-48. DOI: 10.1056/NEJMoa1209096 PMID: 23228172.

7. Ryan CJ, Smith MR, Fizazi K, Saad F, Mulders PF, Sternberg $\mathrm{CN}$, et al. Abiraterone acetate plus prednisone versus placebo plus prednisone in chemotherapy-naive men with metastatic castration-resistant prostate cancer (COU-AA-302): final overall survival analysis of a randomised, double-blind, placebo-controlled phase 3 study. Lancet Oncol. 2015;16(2):152-60. DOI: 10.1016/S14702045(14)71205-7 PMID: 25601341.

8. Uluocak N, Parlaktas BS, Deniz FE, Erdemir F, Koseoglu RD, Gedar MO. Orbital metastasis of prostate cancer: a case report. Kaohsiung J Med Sci. 2007;23(4):199-202. DOI: 10.1016/s1607-551x(09)70398-5 PMID: 17395569.

9. Long MA, Husband JE. Features of unusual metastases from prostate cancer. Br J Radiol. 1999;72(862):933-41. DOI: $10.1259 /$ bjr.72.862.10673942 PMID: 10673942.

10. Lefresne S, Fairchild A, Johnson R, Deschenes J, Russell $\mathrm{L}$, Pederson J. Genitourinary malignancy presenting as an ocular metastasis: A case report and review of the literature. Can Urol Assoc J. 2012;6(2):E67-71. DOI: 10.5489/ cuaj.10174 PMID: 22511437.

11. Boldt HC, Nerad JA. Orbital metastases from prostate carcinoma. Arch Ophthalmol. 1988;106(10):1403-8. DOI: 10.1001/archopht.1988.01060140567024 PMID: 3178551.

12. Patel AR, Olson KB, Pienta KJ. Proptosis and decreased vision secondary to prostate cancer orbital wall metastasis. Anticancer Res. 2005;25(5):3521-2. PMID: 16101173.

13. Hoffmann MA, Wieler HJ, Baues C, Kuntz NJ, Richardsen I, Schreckenberger M. The Impact of 68Ga-PSMA PET/CT and PET/MRI on the Management of Prostate Cancer. Urology. 2019;130:1-12. DOI: 10.1016/j.urology.2019.04.004 PMID: 30986486.

14. Cornford P, Bellmunt J, Bolla M, Briers E, De Santis M, Gross T, et al. EAU-ESTRO-SIOG Guidelines on Prostate Cancer. Part II: Treatment of Relapsing, Metastatic, and Castration-Resistant Prostate Cancer. Eur Urol. 2017;71(4):630-42. DOI: 10.1016/j.eururo.2016.08.002 PMID: 27591931.

15. Pastor Navarro H, Donate Moreno MJ, Carrion Lopez P, Martinez Ruiz J, Lorenzo Romero JG, Jimenez Bachs JM, et al. [Retroorbital metastasis in the initial diagnosis of prostate cancer]. Arch Esp Urol. 2008;61(4):524-7. DOI: 10.4321/s0004-06142008000400010 PMID: 18592772. 